\title{
A REVIEW ON DATA COLLECTION USING MOBILE NODES IN WSN
}

\author{
Chamanpreet Kaur (1), Vikramjit Singh(2) \\ (1) Research Scholar, Department of Computer Science and Engineering, NWIET, Moga \\ (2) Assistant Professor, Department of Computer Science and Engineering, NWIET, Moga
}

\begin{abstract}
Wireless sensor networks have become increasingly popular due to their wide range of application. Clustering sensor nodes organizing them hierarchically have proven to be an effective method to provide better data aggregation and scalability for the sensor network while conserving limited energy. Minimizing the energy consumption of a wireless sensor network application is crucial for effective realization of the intended application in terms of cost, lifetime, and functionality. However, the minimizing task is hardly possible as no overall energy cost function is available for optimization. The need for energyefficient infrastructures for sensor networks is becoming increasingly important. Wireless sensor networks are networks consisting of many sensor nodes that communicate over a wireless media. A sensor node is equipped with a sensor module, a processor, a radio module and a battery. Since the battery limits the lifetime of the sensor nodes it also limits the lifetime of the sensor network, thus energy efficiency is a major issue for sensor networks. An important goal in many sensor networks is to monitor an area as long time as possible. Hence, it is important to distribute energy consumption evenly across the network. When the energy consumption is evenly distributed, the major part of the sensor nodes will stay alive approximately equally long time.
\end{abstract}

\section{Keywords}

Wireless Sensor Network, Energy saving, Protocol, Cluster Head, Node, WSN.

\section{INTRODUCTION}

A Wireless Sensor Network or WSN is supposed to be made up of a large number of sensors and at least one base station. The sensors are autonomous small devices with several constraints like the battery power, computation capacity, communication range and memory. They also are supplied with transceivers to gather information from its environment and pass it on up to a certain base station, where the measured parameters can be stored and available for the end user. In most cases, the sensors forming these networks are deployed randomly and left unattended to and are expected to perform their mission properly and efficiently. As a result of this random deployment, the WSN has usually varying degrees of node density along its area. Sensor networks are also energy constrained since the individual sensors, which the network is formed with, are extremely energy-constrained as well. The communication devices on these sensors are small and have limited power and range. Both the probably difference of node density among some regions of the network and the energy constraint of the sensor nodes cause nodes slowly die making the network less dense. Also, it is quite common to deploy WSNs in harsh environment, what makes many sensors inoperable or faulty. For that reason, these networks need to be fault-tolerant so that the need for maintenance is minimized. Typically, the network topology is continuously and dynamically changing, and it is actually not a desired solution to replenish it by infusing new sensors instead the depleted ones. A real and appropriate solution for this problem is to implement routing protocols that perform efficiently and utilizing the less amount of energy as possible for the communication among nodes. A wireless sensor network is a technology that emerges as a consequence of the evolution of network technology along with microelectronics and micromechanical devices. It is a new concept, a view towards the future, a clear consequence of the new steps forward in the communications field. In few words a wireless sensor network, is a network that could contain from a couple to many small nodes with sensors attached and communications capabilities to transmit and receive information. The data acquired by these sensors is then transmitted along a network from one node to another until it is collected in a central unit, normally connected to a PC. If the original expectations created around this new network concept come true, it will improve monitoring and control systems used nowadays in the environment, medical, industry, consumer, and military sectors.

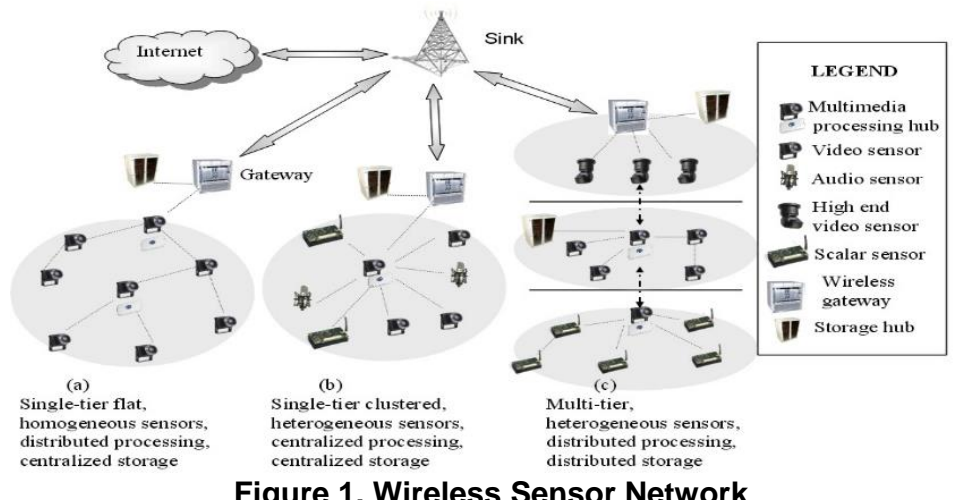

With the help of this technology such systems will now be capable of raising a huge amount of data and results which will be available for analysis in real time. This could lead to a new era of monitoring and controlling processes as they are occurring, something that it was not possible before in some cases without the intervention of persons and complex 
equipment. Another advantage in the use of wireless technology is the reduction in cost that cabling deployment has in current systems and also the possibility of performing measurements in inaccessible places. In ad-hoc network each and every nodes are allow to communicate with each other without any fixed infrastructure. This is actually one of the features that differentiate between ad-hoc and other wireless technology like cellular networks and wireless LAN which actually required infrastructure based communication like through some base station. [2].Wireless sensor network is one of the category belongs to ad-hoc networks. Sensor network are also composed of nodes. Here actually the node has a specific name that is "Sensor" because these nodes are equipped with smart sensors [2]. A sensor node is a device that converts a sensed characteristic like temperature, vibrations, pressure into a form recognize by the users. Wireless sensor networks nodes are less mobile than ad-hoc networks. So mobility in case of ad-hoc is more. In wireless sensor network data are requested depending upon certain physical quantity. So wireless sensor network is data centric. A sensor consists of a transducer, an embedded processor, small memory unit and a wireless transceiver and all these devices run on the power supplied by an attached battery [1].

The WSN consist of two main components:

1. Sensor Nodes, and

2. Base Station (Central Gateway)

\section{SENSOR NODES}

Sensors nodes are typically built of few sensors and a mote unit as shown in Fig.1.2.A Sensor is a device which senses the information and pass it on to mote. Sensors are typically used to measure the changes in physical environmental parameters like temperature, pressure, humidity, sound, vibration and changes in the health parameter of person e.g. blood pressure and heartbeat. MEMS based sensor have found good use in sensor nodes. A mote consists of processor, memory, battery, $A / D$ converter for connecting to a sensor and a radio transceiver for forming an ad hoc network. A mote and sensor together form a Sensor Node. A sensor network is a wireless ad-hoc network of sensor nodes. Each sensor node can support a multi-hop routing algorithm and function as forwarder for relaying data packets to a base station.

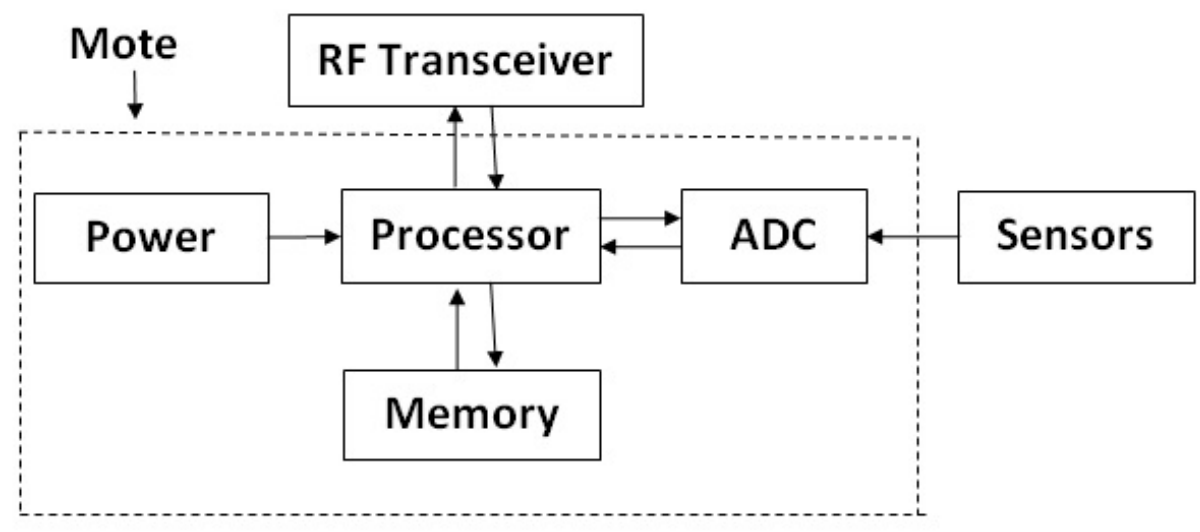

Figure 2. Block Diagram of Sensor Node

\section{BASE STATION}

A base station links the sensor network to another network. It consists of a processor, radio board, antenna and USB interface board. It is preprogrammed with low-power mesh networking software for communication with wireless sensor nodes. Deployment of the base station in a wireless sensor network is very important as all the sensor nodes handover their data to the base station for processing and decision making. Energy conservation, coverage of sensor nodes and reliability issues are taken care of during deployment of base station in sensor network. Generally base stations are assumed static in nature but in some scenarios they are assumed to be mobile to collect the data from sensor nodes.

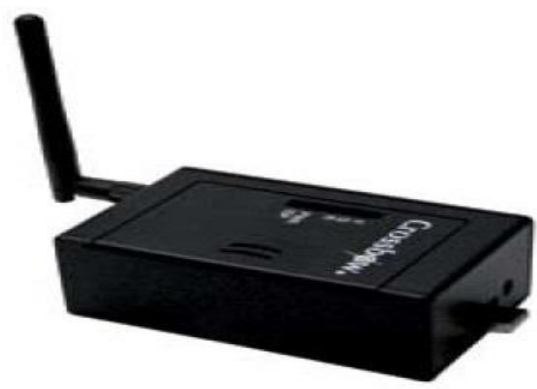

Figure 3. Base Station 


\section{RADIO MODEL}

We have assumed the same radio model which has been used in earlier works. For the radio hardware, the transmitter dissipates energy to run the transmitter radio electronics and power amplifier, and the receiver dissipates energy to run the receive radio electronics as shown in Figure 4 . For the scenarios described in this project work, both the free space $\left(\mathrm{d}^{2}\right.$ power loss) and the multi path fading ( $\mathrm{d}^{4}$ power loss) channel models were used depending on the distance between the transmitter and the receiver. If the distance is less than a threshold, the free space $\left(f_{s}\right)$ model is used; otherwise, the multi path $\left(m_{p}\right)$ model is used.

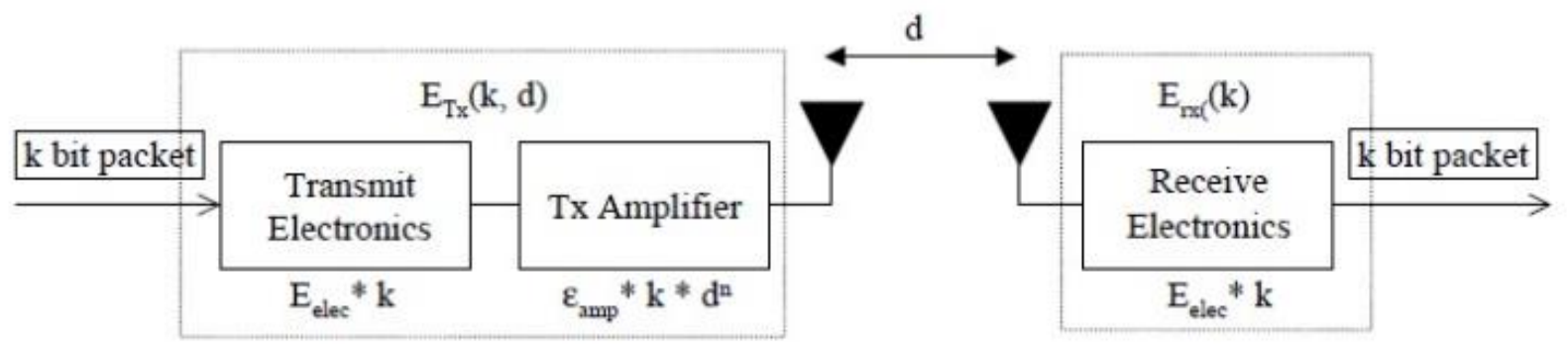

Figure 4. Radio Model

\section{APPLICATIONS OF WIRELESS SENSOR NETWORKS}

Sensor networks are applied in a wide range of areas, such as military applications, public safety, medical, surveillances, environmental monitoring, commercial applications, habitat and tracking. In general, sensor networks are ubiquitous since they support opportunities for the interaction between humans and their physical world. In addition, sensor networks are expected to contribute significantly to pervasive computing and space exploration. Moreover, other applications for wireless sensor networks can be seen in environmental monitoring and control field, high-security smart homes, tracking, and identifications and personalization.

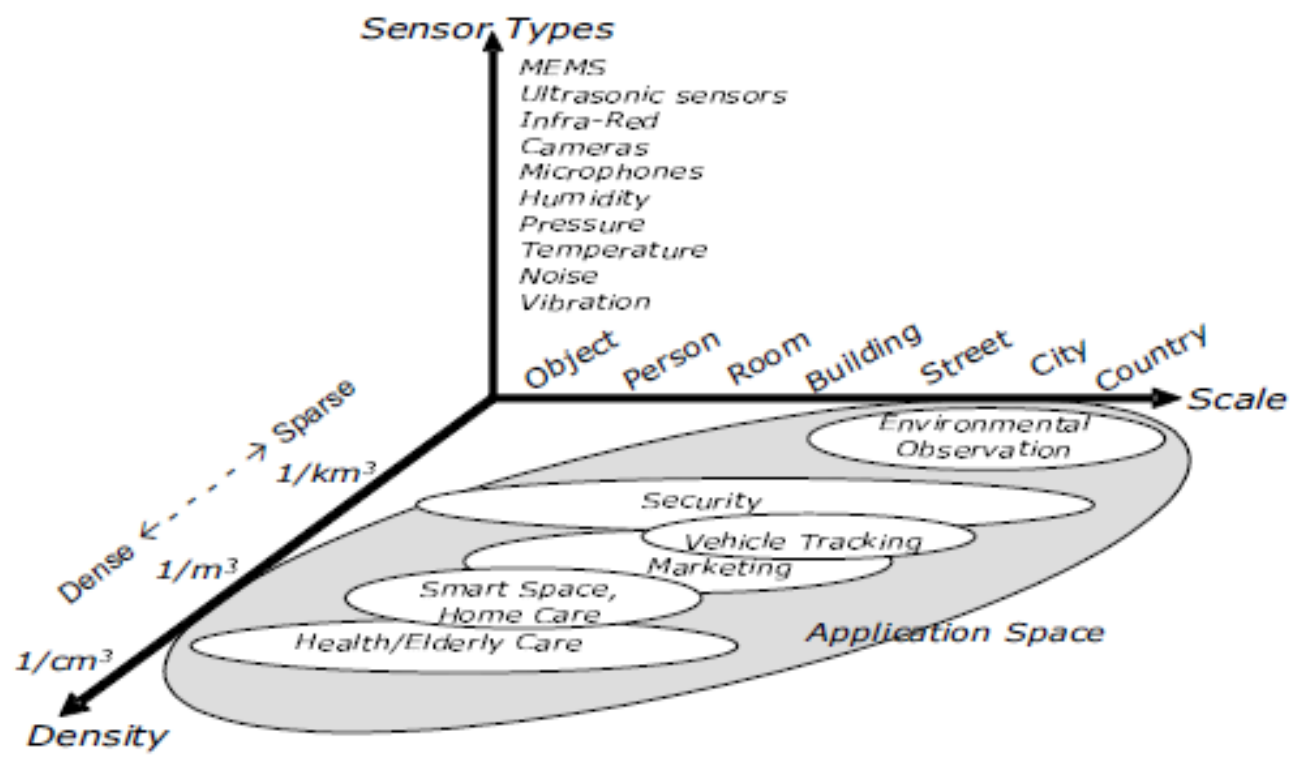

Figure 5. Application Space

> Military Applications: Wireless sensor networks are becoming an integral part of military command, control, communication, computing, intelligence, surveillance and targeting systems. The rapid deployment, fault tolerance and self-organization characteristics of sensor networks make them a very promising sensing technique for military. Since sensor networks are based on dense deployment of disposable and low cost sensor nodes, destruction of some nodes by hostile actions does not affect military applications as much as the destruction of traditional sensor, which makes sensor networks concept a better approach for battlefield. Various military applications of sensor networks are monitoring friendly forces, equipment's and ammunition; biological and chemical (NBC) attack detection and reconnaissance [4].

$>$ Environmental Applications: Some environmental applications of sensor network include tracking the movement of birds , small animals and insects; monitoring environmental conditions that affect crops and livestock ; irrigation; macro instruments for large scale earth monitoring and planetary exploration; chemical/bio logical detection; precision 
agriculture; biological, Earth and environmental monitoring in marine, soil and atmospheric contexts ; forest fire detection and meteorological an d geophysical research; flood detection; bio complexity mapping of the environment and pollution study [4].

> Health Application: Some of the applications are providing interfaces for the disabled; integrated patient monitoring; diagnostics; drug administration in hospital; monitoring the movements and internal process of insects or others mall animals; tele-monitoring of human physiological data; and tracking and monitoring doctors and patients inside a hospital. For example, sensors, can be deployed in a patient's home to monitor the behaviors of the patient. It can alert doctors when the patient falls and requires immediate medical attention [4].

> Home Applications: Wireless sensor networks can be used to provide more convenient and intelligent living environments for human beings. Home automation; as technology advances, smart sensor nodes and actuators can be buried appliances, such as vacuum cleaners, microwave ovens, refrigerators and VCRs. These sensor nodes inside the domestic devices can interact with each other and with an external network via the internet or satellite. They allow end users to manage home devices locally and remotely more easily.

> Agriculture using wireless sensor networks within the agricultural industry is increasingly common; using a wireless network frees the farmer from the maintenance of wiring in a difficult environment. Gravity feed water systems can be monitored using pressure transmitters to monitor water tank levels, pumps can be controlled using wireless I/O devices and water use can be measured and wirelessly transmitted back to a central control center for billing. Irrigation automation enables more efficient water use and reduces waste [5].

$>$ Structural Monitoring Wireless sensors can be used to monitor the movement within buildings and infrastructure such as bridges, flyovers, embankments, tunnels etc. enabling Engineering practices to monitor assets remotely without the need for costly site visits, as well as having the advantage of daily data, whereas traditionally this data was collected weekly or monthly, using physical site visits, involving either road or rail closure in some cases. It is also far more accurate than any visual inspection that would be carried out [5].

\section{CLUSTERING IN WIRELESS SENSOR NETWORKS}

Despite the innumerable applications of WSNs, these networks have several restrictions, e.g., limit deanery supply, limited computing power, and limited bandwidth of the wireless links connecting sensor nodes. Wireless sensor networks are energy-limited and application-specific. To reduce the cost and size of these sensors, they are equipped with small batteries. Each battery-power sensor node is a constrained device with a relatively small memory resources, restricted computational power, and limited communication capability. A sensor node in such a network can interact directly only with other sensors lying within a small range. To achieve communication within sensors out of each other's communication range, the sensors form a multi-hop communication network [3]. While concentrating on the general network design issues in wireless sensor networks, there are numerous vital aspects that need attention such as the small size of the sensor node, its hardware complexity and ultra-low energy consumption. Among them, energy efficiency is considered as the key design objective, since a sensor node can only be furnished with a limited energy supply.

Clustering is one of the design methods used to manage the network energy consumption efficiently, by minimizing the number of nodes that take part in long-distance communication with the base station and distributing the energy consumption evenly among the nodes in the network. In Clustering, the sensor nodes are apportioned into distinctive groups. Each one cluster is overseen by a node alluded as group head $(\mathrm{CH})$ and different nodes are alluded as cluster nodes. Group nodes don't speak straightforwardly with the sink node. They need to pass the gathered information to the cluster head. Group head will total the information, gained from cluster nodes and transmits it to the base station. In this way it minimizes number of messages imparted to base station.

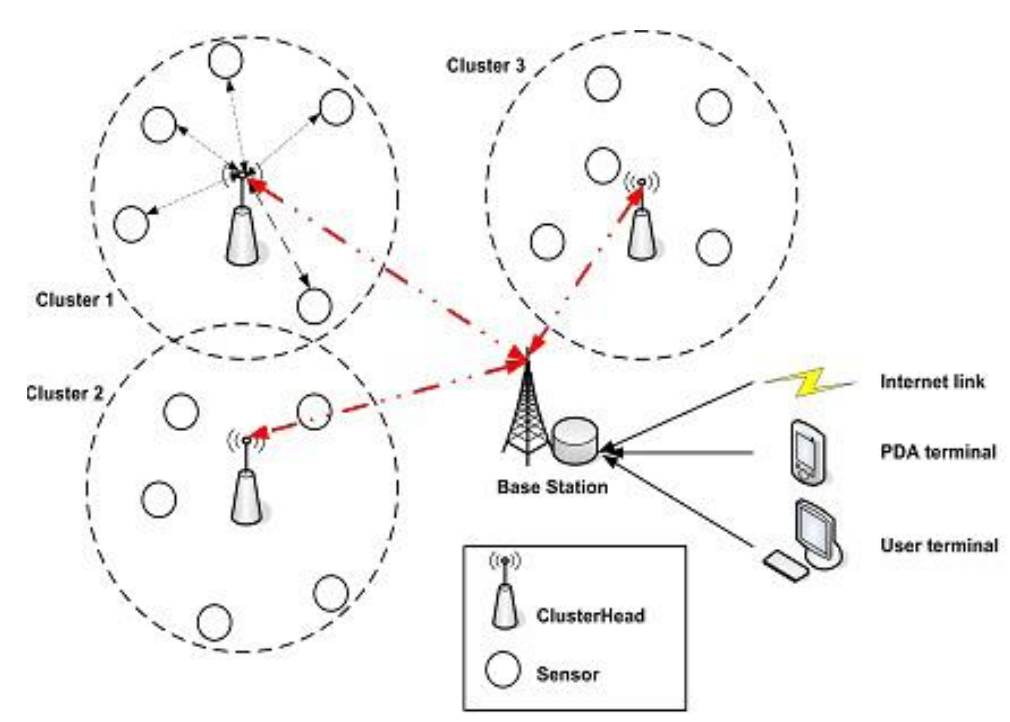

Figure 6. Clustering in WSN 


\section{CLUSTERING PARAMETERS}

There are some basic parameters regarding clustering procedures in WSN.

1. Intra-cluster communication: In few initial clustering approaches, a sensor and its designated $\mathrm{CH}$ are assumed to be communicating directly known as one-hop communication. However, multi-hop intra-cluster communication is often required in case when the number of sensor nodes is very large and the number of $\mathrm{CHs}$ is bounded or the communication range of the sensor nodes is limited.

2. Nodes and $\mathrm{CH}$ mobility: The network of stationary sensor nodes and stationary $\mathrm{CHs}$ usually leads to stable clusters with facilitated intra-cluster and inter-cluster network management. While, in case of mobile $\mathrm{CHs}$ or the nodes, there should be dynamic change of cluster membership for each node; subsequently forcing clusters to evolve over time and continuously maintained.

3. Cluster formation methodology: Most often when there is much concern for time efficiency, in that case clustering is done in a distributed manner without coordination. While in few earlier approaches, a centralized (or hybrid) approach was much preferred and followed; one or more coordinator nodes are used to partition the whole network off-line and control the cluster membership.

4. Cluster-head selection: The leader nodes of the clusters in some algorithms particularly for heterogeneous environments can be pre-assigned. In homogeneous environments, the CHs are selected from the deployed set of nodes either based on a specific criterion (residual energy, connectivity etc.) or any probability or completely random way.

5. Multiple levels. Several studies illustrate that instead of one cluster level, the concept of a multi-level cluster hierarchy achieves even better energy distribution and total energy consumption. The improvements offered by multi-level clustering are under research and exploration for very large networks and inter- $\mathrm{CH}$ communication efficiency is of high importance.

\section{RELATED WORK}

The needed detailed literature survey, to get preliminary knowledge and search scope of investigation, to implement Low energy adaptive clustering hierarchy, is explained in this chapter. This synopsis presents investigational studies in several energy efficient routing algorithms and its general purpose.

Yu Xiuwu et al (2016) proposes a routing protocol that is based on monitoring area partition clustering for energy-balanced (EBPC). They divided monitoring area into several virtual partitions, controlled the number of common nodes and clusters by data fusion rate, and forwarded cluster heads data to the next partitions with minimum path selection coefficient.

Samaresh Bera et al (2016) proposes a software-defined wireless sensor network architecture (Soft-WSN)—an effort to support application-aware service provisioning in Internet of Things (IOT). Detailed architecture of the proposed system is presented involving the application, control, and infrastructure layers to enable software-defined networking (SDN) in loT.

Alduais et al (2016) specifies that WSN has become an enabler technology for the IOT applications which extend the physical reach of the monitoring capability. WSN as it is, possess several constraints such as limited energy availability, low memory size, and low processing speed, which are the principal obstacles to designing efficient management protocols for WSNs, what more if it concerns WSN-IOT integration.

Omkar Singh et al (2016) compares three well accepted WSN routing protocols namely LEACH, FAIR and SEP for their energy pattern in heterogeneous scenario. Heterogeneity is introduced in terms of initial random energy values given to the sensor nodes. All simulations are done in MATLAB. Different parameters are used for checking the efficacy of the considered routing protocol for H-WSN.

Rani Kumari et al (2016) analyzes the performance of the various routing protocols of the wireless network in the WBAN and WSN. Moreover, the paper also compares their performance in the same network and in different network using different parameters i.e. PDR, latency and throughput etc. The research signifies that the wireless protocols needs updating to perform well in the WBAN.

Hector Kaschel et al (2016) describes the mechanisms to achieve energy efficiency in a WSN, focusing particularly on routing protocols. Based on the state of the art, is presented a complete taxonomy of routing protocols used in WSN exposing their level of energy efficiency.

Asdianur et al (2016) studied effectiveness in using Compressive Sensing (CS) algorithm in order to reduce measuring in IEEE 802.15.4 Standard Wireless Sensor Network (WSN). As well known, in common WSN work system, Base Station (BS) gather some information from available nodes, which the process itself consumes a lot of energy from each node.

Mohd Zaki Shahabuddin et al (2016) proposes fundamental modelling of topology control algorithm to conserve individual WSN node's energy, and at the same time preserving the graph connectivity. The proposed topology control algorithm consists of three phases: 1. Identifying connecting nodes at maximum transmission, 2. pairing nodes with shortest algorithm/minimum energy level, 3. Calculating/setting minimum power transmission per-node for energy conservation. The algorithm works-out locally and dispenses full graph connectivity, and theoretically would be able to reduce WSN control overhead.

Abhaykumar et al (2016) presents a novel scheme to improve the lifetime of a sensor node by optimizing the size of the packet such that there is no loss of data along with providing an increased lifetime for the nodes. Wireless sensor network is a rapid developing area with diverse applications. Smartness and interoperability of network keeps it in high demand and 
hence comes need for the efficiency of the system. The most important limitation on sensor node is the low power consumption. Sensor nodes carry inadequate, generally irreplaceable power sources. Therefore, while traditional networks aim to achieve high quality of service (QoS), wireless sensor network protocols must emphasis predominantly on power conservation.

B. Bengherbia et al (2016) states that the wireless sensor network (WSN) represent fertile area for research and exploration, especially with enormous development in modern electronic systems, where it becomes an alternative to traditional wired systems. The wireless stems are less energy-consumptive and inexpensive, in addition it helps to reduce the complexity of wiring. Wireless sensor networks require the design of high density computing, an energy-efficient and a flexible node architecture.

Gagandeep Kaur et al (2016) decreases the passive attack on sink node by decreasing the traffic on sink node. The simulation results demonstrate the proposed method can each node will compress their data before sending to cluster head. After compressing, the packet size of node will decrease. This will decrease the traffic overload.

Mei Wu et al (2016) proposes a new model to efficiently control cluster-heads using heterogeneous sensors in real-time. It also proposes a generic software framework to feasibly and efficiently meet the needs of the users and applications (e.g., reliability, energy efficiency). The proposed model and its method have been implemented and evaluated in MATLAB, comparing its performance with known protocols for WSNs. The proposed model is suitable for common use because it is superior to other methods by energy efficiency.

Sanaa. S et al (2016) introduces an authentication protocol and simple key distributed scheme between sensor nodes. Node mobility has been taken into consideration and the work proposes a re-authentication protocol that is very efficient than the initial protocol. A wireless sensor network (WSN) consists of thousands of sensors and one base station. Sensors are deployed in the network to monitor target area and sense information according to the applied application then send this information to the base station.

Roman Lara-Cueva et al (2016) presents a study in order to identify the value range of the main parameters within Carrier Sense Multiple Access (CSMA) defined in IEEE 802.15.4 that guarantee a satisfactory Wireless Sensor Networks (WSN) performance for a possible volcano monitoring application. Moreover, this study performs the comparison between test-bed in outdoor scenarios with the purpose of distinguishing the optimal number of nodes or each gateway according the main constrains given by an existing sensor network for real-time (RT) volcano monitoring system such as sampling time, packet loss, and delay.

\section{RESEARCH GAP}

Due to the deployment of WSNs in unattended harsh environment conditions, it gets to be unrealistic to charge or replace their batteries. Accordingly, the energy efficient operation of wireless sensors is very important to prolong the lifetime of overall wireless sensor networks. Furthermore, in WSN, there is wireless communication among all the nodes. Moreover, the energy consumed to transmit a message is exceptionally more than the energy needed to receive the same message. Besides all this, the route of each message destined to the base station too decides the network lifetime; for example, any node with depleted battery in short routes to the base station may lead to decreased network lifetime. While, using a long route composed of many sensor nodes can significantly increase the network delay.

- To decide cluster area on the basis of chosen cluster head consumes huge amount of energy resources in the communication between nodes for the purpose of fixing the cluster region and its head. When two cluster heads are very close to each other that means their ranges will overlap each other which will create a major problem.

- No parameter has been explained for the election of Cluster head. There are several parameters that are involved in the election of cluster head.

- $\quad$ Same level of power is used to amplify the signals from member node to cluster head, cluster head to mobile node and mobile node to base station. This will consume lot of energy.

- Mobile node requires large amount of energy for transferring the data of complete sector to the base station.

\section{MATLAB}

MATLAB is a programming language developed by Math Works. It started out as a matrix programming language where linear algebra programming was simple. It can be run both under interactive sessions and as a batch job. MATLAB is a highperformance language for technical computing and interactive environment that integrates computation, visualization, and programming in an easy-to-use environment where problems and solutions are expressed in familiar mathematical notation. One can solve technical computing problems faster than with traditional programming languages, such as $\mathrm{C}, \mathrm{C}++$, and FORTRAN with use of MATLAB. MATLAB provides a number of features for documenting and sharing of work. One can integrate his/her MATLAB code with other languages and applications, and distribute their MATLAB algorithms and applications.

Some features of MATLAB include:

- High-level language for technical computing

- Development environment for managing code, files, and data

- Interactive tools for iterative exploration, design, and problem solving

- Mathematical functions for linear algebra, statistics, Fourier analysis, filtering, optimization, and numerical integration

- 2-D and 3-D graphics functions for visualizing data 
- Tools for building custom graphical user interfaces

\section{CONCLUSION}

Due to recent technological advances, the manufacturing of small and low cost sensors became technically and economically feasible. The sensing electronics measure ambient conditions related to the environment surrounding the sensor and transforms them into an electric signal. Processing such a signal reveals some properties about objects located and/or events happening in the vicinity of the sensor. A large number of these disposable sensors can be networked in many applications that require unattended operations. A Wireless Sensor Network (WSN) contain hundreds or thousands of these sensor nodes. These sensors have the ability to communicate either among each other or directly to an external base station. The need for energy-efficient infrastructures for sensor networks is becoming increasingly important. Wireless sensor networks are networks consisting of many sensor nodes that communicate over a wireless media. A sensor node is equipped with a sensor module, a processor, a radio module and a battery. Since the battery limits the lifetime of the sensor nodes it also limits the lifetime of the sensor network, thus energy efficiency is a major issue for sensor networks. An important goal in many sensor networks is to monitor an area as long time as possible. Hence, it is important to distribute energy consumption evenly across the network. When the energy consumption is evenly distributed, the major part of the sensor nodes will stay alive approximately equally long time. In this paper, we have reviewed the energy saving mechanisms and stated few problems among them.

\section{REFERENCES}

[1] Y. Xiuwu, Fan Feisheng Zhou Lixing and Z. Feng, "WSN Monitoring Area Partition Clustering Routing Algorithm for Energy-Balanced," IEEE, pp. 80-84, 2016.

[2] S. Bera, S. Misra, Sanku Kumar Roy and Mohammad S. Obaidat, "Soft-WSN: Software-Defined WSN Management System for loT Applications," IEEE, pp. 1-8, 2016.

[3] N. A. M. Alduais, J. Abdullah, J. Abdullah, A. Jamil and L. Audah, "An Efficient Data Collection and Dissemination for IOT based WSN," IEEE, 2016.

[4] O. Singh, V. Rishiwal and M. Yadav, "Energy Trends of Routing Protocols for H-WSN," IEEE, 2016.

[5] R. Kumari and. P. Nand, "Performance Comparison of various Routing Protocols in WSN and WBAN," IEEE, pp. $427-$ 431, 2016.

[6] Hector Kaschel and ohanna Ortega , "Energy efficiency in routing protocols applied to WSN," IEEE, 2016.

[7] Asdianur Hadi and Ida Wahidah, "Delay Estimation using Compressive Sensing on WSN IEEE 802.15.4," IEEE, pp. 192197, 2016.

[8] Mohd Zaki Shahabuddin, Halabi Hasbullah and Izzatdin A Aziz, "eliminary Framework of Topology Control Algorithm Ahieve Node's Energy Efficiency," IEEE, pp. 259-263, 2016.

[9] Abhaykumar L. Gupta and Narendra Shekokar, "A Novel Approach to Improve Network Lifetime in WSN by Energy Efficient Packet Optimization," IEEE, 2016.

[10] B. Bengherbia, S. Chadli, M. Ould Zmirli and A. Toubal, "A MicroBlaze Based WSN Sink Node Using XBee Transceiver," IEEE, pp. 831-834, 2016.

[11] Gagandeep Kaur, Deepali and Rekha Kalra, "Improvement and Analys Security of WSN From Passive Attack," IEEE, pp. 4520-425, 2016.

[12] M. Wu, H. Liu and Q. Min, "Lifetime Enhancement by Cluster Head Evolutionary Energy Efficient Routing Model for WSN," IEEE, pp. 545-548, 2016.

[13] Roman Lara-Cueva, Rodolfo Gordillo, Liliana Valencia and Diego S. Ben, "Determining the Main CSMA Parameters for Adequate Performance of WSN for Real-time Volcano Monitoring System Applications," IEEE, pp. 1-9, 2016.

[14] Sanaa. S. Abd El dayem and M. R. M. Rizk , "An Efficient Authentication Protocol and Key Estashment in Dynamic WSN," IEEE, pp. 178-182, 2016.

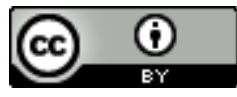

This work is licensed under a Creative Commons Attribution 4.0 International License.

DOI:10.24297/ijct.v16i5.6263 